\title{
Grand challenge: investigating the oxidant hypothesis in the realm of systems physiology and disease pathogenesis
}

\author{
Arturo J. Cardounel* \\ Department of Physiology and Functional Genomics, University of Florida College of Medicine, Gainesville, FL, USA \\ *Correspondence: cardounel.1@ufl.edu
}

Despite significant advancement in our understanding of the role of oxidative stress in disease pathogenesis, the majority of clinical trials examining antioxidant therapy and redox modifying interventions have failed to establish clinical benefit. The lack of clinical efficacy in human trials despite a wealth of literature demonstrating a clear pathogenic role of free radicals in disease states ranging from cardiovascular disease to cancer belies the need for research efforts into the role of oxidants in physiology. With the advent of new free radical detection techniques, advances in molecular biology and the development of in vivo free radical imaging modalities, the field is uniquely positioned to begin to unravel the molecular mechanisms of free radical injury and redox signaling within the realm systems physiology and disease pathogenesis. In this regard, the challenges we face lie in our ability to reconcile past discovery and current knowledge with future efforts to examine whether the oxidative modification hypothesis is relevant to human disease. The challenges begin with identifying viable therapeutic targets and approaches to restore redox balance and ameliorate free radical injury and the physiological disturbances resulting from oxidative stress.

As a result of a large body of epidemiological data showing a protective role for dietary antioxidants, numerous clinical trials have been initiated to examine the benefit of supplementation with dietary antioxidants primarily focusing on vitamin $E$, vitamin $C$, or $B$-carotene (Jha et al., 1995). Unfortunately, in the majority of studies, antioxidants are looked on as a class of compounds sharing certain common properties that are functionally considered interchangeable. There may be some justification for such classification when dealing with simple in-vitro redox systems, however, there are striking differences with regards to there chemical properties. For example, $B$-carotene is an excellent scavenger of singlet oxygen but much less effective at terminating free radical chain reactions; the reverse is true of vitamin E (Packer et al., 1981). In the context of a complex biological system, such aggregate classification becomes hard to defend. For example, vitamin $\mathrm{C}$ is watersoluble, readily absorbed, and transported in the aqueous phase of the plasma; vitamin E is lipophilic, poorly absorbed in the GI tract, and transported with plasma proteins (Padayatty et al., 2004; Rigotti, 2007). The pharmacodynamics of the various antioxidants also differ greatly, and until we know where and how these agents function, we have no way to predict which antioxidant, at what dosage and administered by what route, will be most effective. An analysis of the hypothesis and the trial literature, points out major misconceptions about the hypothesis and unjustified outcome expectations of antioxidant trials. In response, future efforts in the field of oxidant physiology should include: (1) identification of novel and selective biochemical and biological markers of oxidative stress; (2) informed antioxidant selection based on comprehensive pharmacokinetic profiles and identification of physiological targets of action; and (3) establishment of selection criteria for patient recruitment.

Through their effects on proteins, DNA and membranes, oxidizing and reducing agents play a critical role in regulating a myriad of cellular functions. Our goal is to publish research related to furthering the understanding of the integrative mechanisms of redox signaling in the control of complex physiological function. This aspect is critical to our ability to understand, manage and treat diseases associated with oxidant stress and impaired antioxidant defenses. Because of the integrative nature of the specialty section, the scope of articles that will be considered is broad, and includes work elucidating genetic, biophysical, genomic, proteomic, cellular, molecular, biochemical, physiological and pharmacological aspects of oxidants and redox active molecules, as well as the pathological events associated with oxidants stress. Free radical imaging studies and clinical studies targeted to mechanistic aspects of oxidative stress are also welcome.

In conclusion, new technologies and animal models of disease will continue by ingenious innovations to make inroads in our understanding of the role oxidants play in physiology and medicine. The use of novel molecular, genetic and imaging approaches will undoubtedly provide fundamental advances in the realm of oxidant physiology. This is the good news! The bad news is that we need to wait to find out how.

\section{REFERENCES}

Jha, P., Flather, M., Lonn, E., Farkouh, M., and Yusuf, S. (1995). The antioxidant vitamins and cardiovascular disease. A critical review of epidemiologic and clinical trial data. Ann. Intern. Med. 123, 860-872.

Packer, J. E., Mahood, J. S., Mora-Arellano, V. O., Slater, T. F., Willson, R. L., and Wolfenden, B. S. (1981). Free radicals and singlet oxygen scavengers: reaction of a peroxy-radical with beta-carotene, diphenyl furan and 1,4-diazobicyclo (2,2,2)-octane. Biochem. Biophys. Res. Commun. 98, 901-906.

Padayatty, S. J., Sun, H., Wang, Y., Riordan, H. D., Hewitt, S. M., Katz, A., Wesley, R. A., and Levine, M. (2004). Vitamin C pharmacokinetics: implications for oral and intravenous use. Ann. Intern. Med. 140, 533-537.

Rigotti,A. (2007).Absorption, transport, and tissue delivery of vitamin E. Mol. Aspects Med.28, 423-436.

Received: 29March 2010; accepted: 01 April 2010; published online: 05 May 2010.

Citation: Cardounel AJ (2010) Grand challenge: investigating the oxidant hypothesis in the realm of systems physiology and disease pathogenesis. Front. Physio. 1:4. doi: 10.3389/ fphys.2010.00004

This article was submitted to Frontiers in Oxidant Physiology, a specialty of Frontiers in Physiology.

Copyright (c) 2010 Cardounel. This is an open-access article subject to an exclusive license agreement between the authors and the Frontiers Research Foundation, which permits unrestricted use, distribution, and reproduction in any medium, provided the original authors and source are credited. 